\title{
Hybrid Satellite - Wireless Sensor Networks Architecture for Telemedicine Applications in the Context of Emergency Satellite Communications in $\mathrm{Ku} / \mathrm{Ka} / \mathrm{Q} / \mathrm{V}$ Bands
}

\author{
Rahim Kacimi ${ }^{1, *}$, Ponia Pech ${ }^{2}$ \\ ${ }^{1}$ University of Toulouse, IRIT-UPS, 118 route de Narbonne, 31062 Toulouse, France \\ ${ }^{2}$ Independent researcher, formerly at TeSA Association Lab., 14-16, Port Saint-Etienne, 31000 Toulouse, France
}

\section{Abstract}

Thanks to the facilities offered by telecommunications, telemedicine today allows physicians and clinicians to access, monitor and diagnose patients remotely. Telemedicine includes several applications such as remote monitoring of chronically ill patients, monitoring people in their everyday lives to provide early detection and intervention for various types of diseases, computer-assisted physical rehabilitation in ambulatory settings, and assisted living for the elderly at home, as well as remote monitoring of injured people in a postdisaster situation. These new applications require a reliable, wireless communication link between the devices implanted in the patient's skin and a clinician. In this article, this issue is discussed and a list of performance criteria for the different communication links used are addressed, especially focusing on the satellite link. Then an adaptive air interface which is designed to meet the performance constraints of bidirectional satellite communication links in an emergency situation in $\mathrm{Ku} / \mathrm{Ka} / \mathrm{Q} / \mathrm{V}$ bands where when strong channel impairments occur is described and analysed.

Received on 28 February 2014; accepted on 20 December 2014; published on 28 December 2014

Keywords: vital sign monitoring, health-care monitoring, wireless sensor networks, wireless body networks, satellite, telemedicine

Copyright (C) 2014 Rahim Kacimi and Ponia Pech, licensed to ICST. This is an open access article distributed under the terms of the Creative Commons Attribution license (http://creativecommons.org/licenses/by/3.0/), which permits unlimited use, distribution and reproduction in any medium so long as the original work is properly cited.

doi:10.4108/mca.2.5.e1

\section{Introduction}

Nowadays, remote health-care is becoming increasingly attractive due to several advantages it brings: extending the health system coverage to rural and isolated areas, ensuring autonomy to chronic patients by letting them stay at home, allowing the clinicians to remotely diagnose and monitor their patients, ensuring realtime monitoring for critical illnesses, etc. Moreover, advances in key areas such as Wireless Sensor Networks (WSN) and Body Sensor Networks (BSN) are enabling technologies for the application domain of unobtrusive medical monitoring [42]. This field includes cable-free continuous monitoring of vital health signs in intensive care units, remote monitoring of chronically ill patients,

${ }^{*}$ Corresponding author. Email: rahim.kacimi@irit.fr monitoring people in their everyday lives to provide early detection and intervention for various types of diseases, computer-assisted physical rehabilitation in ambulatory settings, and assisted living for the elderly at home, as well as remote monitoring of injured people in a post-disaster situation. These innovative applications, where a pacemaker communicates the patient's health state and performance data to a base station, or a BSN integrating a number of devices, require a reliable wireless communication link between the sensing devices implanted in the patient's skin and a physician. Otherwise, the wireless link can be used to interrogate the implant at either irregular intervals, or on a regular basis, or to provide near permanent communication. A one-way wireless link may be used to obtain the patient's health information or performance data from the implanted device, while 
a two-way link allows external reprogramming of an implanted device [42]. Due to the critical and sensitive nature of the medical information transmitted through the wireless network, reliable data transfer for BSNs and network reliability are of paramount importance. In addition to wireless terrestrial networks, satellite link can (or have to) be used in some cases. For example, when the patient is outside of the cellular network coverage, the satellite network can be used to transmit data to the medical call center or to contact the patient for further information. Network reliability directly affects the quality of the patient's monitoring, and in a worst-case scenario can be fateful when a life threatening event has gone undetected. However, due to the constraints on communication bandwidth and power consumption, traditional network reliability techniques such as the retransmission mechanism for TCP (Transmission Control Protocol) may not be practical for BSN applications, whereas they are used in satellite links despite their drawbacks (as in the URSAFE [17] and OURSES [18] projects that will be described later on below). With similar constraints on WSNs, researchers have proposed several methods for improving their reliability. One simple approach is to use limited retransmission where packets are retransmitted for a fixed number of times until an acknowledgment is received; however, retransmission often induces significant overhead to the network. Another approach is to form a multi-path network and exploit the multiple routes to avoid disrupted links. It is expected that this will be an area that will raise significant research interest in the coming years, particularly in exploring the autonomic sensing paradigm for developing self-protecting, self-healing, self-optimizing, and self-configuring BSNs. Unlike typical wired or wireless network architectures in which the network configuration is mostly static and there are limited constraints on resources, the BSN architecture is highly dynamic, placing more rigorous constraints on power supply, communication bandwidth, storage and computational resources. The remainder of this paper is organized as follows: the context of this work and its critical problems are discussed in section 2. Section 3 states the reliability problem and performance criteria for WBAN (Wireless Body Area Networks) and WSN links. Section 4 focuses on satellite link issues in telemedicine applications. Section 5 thoroughly describes an adaptive DVB-S2-based air interface which is designed to meet the performance constraints of bidirectional satellite communication links in an emergency situation in $\mathrm{Ku} / \mathrm{Ka} / \mathrm{Q} / \mathrm{V}$ bands where when strong channel impairments occur. In section 6 , the main conclusions of our work are summarized, and a set of open issues and future directions is presented.

\section{Context and problem}

TeSA laboratory conducted with its partners two projects in the context of remote health-care. These two projects are UR-Safe (Universal Remote Signal Acquisition For hEalth) and OURSES (Offer of Services using Satellite for Rural Usage). In addition, TeSA also studied a low bit rate adaptive air interface for satellite bidirectional links designed for emergency situations that is suited to post-disaster telemedicine applications, and will be thoroughly presented in section V.

\subsection{UR-Safe project description}

The UR-Safe project [17] aimed at creating a mobile telemedicine care environment for the elderly, thus helping mitigate the problems of health care provision observed in the Western societies caused by an aging population and the associated increasing costs. The adopted technological solution maximizes the concepts of autonomous living and quality of life for the patient, in alignment with the emerging models of health care provision, while at the same time addressing safety and alarm detection issues. The technological solution consists in placing medical sensing devices on the patient's body, all of them being connected via a short range Wireless Personal Area Network (WPAN) to a central, portable electronic unit called Personal Base Station (PBS). These wearable sensors enable to record electrocardiograms (ECG) and oxygen saturation level for instance, while a shock/fall detector sends an alarm when the patient falls or presses a button. Thanks to speech recognition algorithms, the PBS allows the exchange of simple spoken sentences with the patient in order to better analyze the patient's health condition. The pieces of information coming from the different sensors and from the shock/fall detector are gathered. Based on these data, preliminary computeraided diagnosis is performed by the PBS. The data are then sent to a medical call center.

\subsection{OURSES project description}

The OURSES project [18] proposed three telemedicine applications related to services offered to elderly people. It focused on the use of satellites as a complement to terrestrial communication technologies to ensure the deployment of teleservices in areas where telecommunication infrastructure is lacking. These three telemedicine applications are described in the following. A typical architecture of a remote telemedicine solution conjugates tree or four different communication technologies according to different wireless architecture links:

1. The first link: the first link connects the medical nodes and the coordinator, in order to form a WBAN mainly using a star topology. The WBAN 


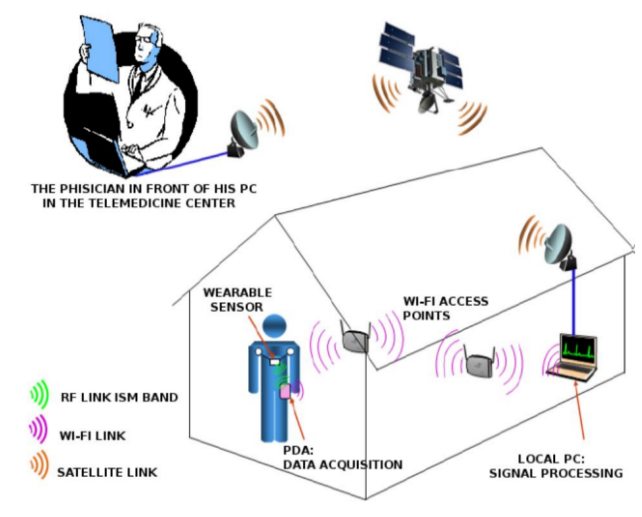

Figure 1. Global scheme of ECG monitoring in OURSES project [18]

is often composed of a limited number of medical nodes (body sensors) connected to a coordinator (that may be a normal sensor node or a PDA (Personal Digital Assistant)).

2. The second link: a second link exists between coordinators and a base station collector (local PC). The routing of information between the coordinators and the base station can be done in various ways and with different communications technologies: on the one hand, a WSN can be formed with all coordinators. The coordinators are also associated with environmental sensors that monitor the physical environment surrounding the patients (temperature, humidity, light,...). The information is then transmitted to the base station via a WSN mesh topology. On the other hand, centralized WiFi technology can replace the WSN by setting direct links between coordinators and access points to collect information.

3. The third link: a last link is used to transmit data from the base station (local PC) to a remote physician. In the absence of wide-coverage terrestrial networks, a satellite link is used in this case.

Figure 1 shows a usage scenario on wireless vital sign monitoring using different technologies. This solution is based on a wireless wearable sensor which transmits ECG to a portable device in real time. The latter is then connected to a local PC which is located in the patient's house through a Wi-Fi link. ECG signals are then sent to the physician's office using a satellite link. When an abnormal event during an ECG analysis is detected by a specific automatic signal-processing-based diagnosis application running on the PC, an alarm is raised and sent to the physician's office.

\subsection{Problem statement}

The purpose of this paper is to join together different scientific communities involved in such challenging projects as telemedicine and biomedical vital signs remote monitoring, namely, the medical end-users (physicians, medical experts), the digital signal processing scientists from academia, and the telecommunication scientists and engineers from both academia and industry. Indeed, telemedicine projects include so wide and multidisciplinary technical and scientific expertise fields that a minimum of dialogue between the different disciplines should be sought for the sake of a better mutual understanding and a more unified and integrated approach. More precisely, one peculiar problem arose in the experiments carried out in the framework of the aforementioned telemedicine projects: packet loss was observed at the physician's PC side on the satellite return channel downlink, which manifested itself in "hole" periods, that is, missing samples in the received ECG signal. In other words, a few ECG samples were missing due to undetected causes occurring somewhere in the wireless/satellite transmission chain that are attributed to the fact that the different links involved (fixed access network, mobile access network and satellite DVBRCS interfacing) are liable to inducing errors or loss on the transmitted data. Such data corruption/loss can occur anytime and anywhere. The timeout of several ARQ (Automatic Repeat Request) procedures was shown to induce packet loss: $1-2 \%$ for GPRS, and $8 \%$ for the satellite [43]. To cope with that problem, TeSA devised a recovering method which hybridized Papoulis-Gerchberg (PG) algorithm and an Auto-Regressive (AR)-based reconstruction algorithm. The principle of the PG exact reconstruction algorithm lies in an interpolation of the missing samples using the band-limited property of the ECG signal, via an iterative process which allows to replace the missing part of the signal with the result of the Inverse Fast Fourier Transform (IFFT). The PG algorithm performs well with a reduced number of missing samples, but its drawback lies in the long convergence time of its iterative process. Therefore another ECG missing sample reconstruction method was proposed, namely, an audio signal reconstruction method based on AR modeling [43]. This method is used to predict forward and backward signal samples. The final step in the proposed reconstruction algorithm is to jointly use the two PG and AR methods, the AR algorithm being executed at initialization phase to be followed by the PG algorithm. The reconstruction performance was measured using the local signal to reconstruction error (noise) ratio given by:

$$
10 \log \left(\frac{\sigma_{x g a p}^{2}}{\sigma_{(x-\hat{x}) g a p}^{2}}\right) \text { in } d B
$$


where $\sigma_{x g a p}^{2}$ represents the variance of the original signal in the missing part and $\sigma_{(x-\hat{x}) g a p}^{2}$ represents the variance of the reconstruction error also restricted in the missing part. Tested on the MIT-BIH Arrhythmia Database, the combined method yielded better performance than the PG and AR algorithms for missing parts of up to 30 consecutive samples $(120 \mathrm{~ms})$. The paper aims at identifying and qualitatively surveying all the different factors related to the wireless and satellite links that may be a cause of packet error or loss, and thus undermine the reconstruction performance of TeSA's algorithm, without any quantitative assessment at this stage. Packet errors and loss can be traced back to mainly three different sources: (i) general performance criteria of the transmission system; (ii) wireless issues; (iii) satellite link issues.

\subsection{Performance criteria}

The performance criteria of wireless network links which have significant implications in the healthcare domain include the number of collisions, the energy consumption at the nodes, the network throughput, the number of unicast packets delivered, the number of packets delivered to each node, the signals received and forwarded to the Medium Access Control (MAC) layer, and the change in energy consumption with variation in transmission range, etc. Some of these criteria are described hereafter:

- Delay: the delay for data packets delivery is of paramount importance in health-care monitoring and its criticality depends on the way data traffic is transmitted. Indeed, the data can be transmitted on either a periodical basis or a nonperiodical basis. A periodic transmission mode corresponds to the case where the traffic data packets collected by the body sensors are sent periodically, while a non-periodic transmission mode refers for instance to the case where an alarm is sent by a body sensor every time it detects an anomaly.

- Packet Received Rate (PRR): in a health-care network solution, a high PRR is necessary so that the physician can make a good diagnostic. For instance, it may sometimes be difficult for him to interpret ECG data, which makes a diagnostic completely impossible.

- Quality of Service (QoS): loss of data is more significant in BSNs, and may require additional measures to ensure QoS and real-time data interrogation capabilities.
3. Wireless sensor and body area networks for vital signs monitoring

The advent of smart wireless sensors that are able to form a BSN would not be possible without the availability of appropriate and inexpensive low power short-range transceivers for low to moderate data rates. These are capable of transmitting real-time data with a latency of typically less than one second within a range of up to five meters.

Current standardization efforts affect most of the layers of a communication stack, starting from the Physical (PHY) layer, including the Medium Access Control (MAC) layer and reaching the higher layers, such as network or routing layers, and even sometimes the data representation and application layers. Different standardization bodies may work in a cooperative fashion, as is the case with ZigBee and IEEE 802.15.4.

The problems encountered in BSNs involved respectively on the first and the second links listed above are summarized as follows:

- Energy consumption: It is widely recognized that limiting energy is an inescapable issue in the design of wireless BSNs due to the strict constraints which it imposes on the network operations. In fact, the energy consumption is a crucial factor impacting the network lifetime that has become the prevailing performance criterion in this area. If the network is to operate as long as possible, these energy constraints require make a trade-off between various activities at both the node and network levels, so that the less energy consumed by the nodes, the longer the network lifetime to satisfy the running application [15].

- Scalability: Scalability is an important factor in designing efficient WSN solutions. A good solution has to be scalable in the sense of being adaptable to future changes in the network topology. Thus scalable protocols should perform well as the network grows larger or as the workload increases.

- Congestion: In healthcare WSN applications (particularly for medical emergencies or closely monitoring critically ailing patients), it is obviously desirable in the first place to avoid congestion, and should it occur, to reduce data loss due to congestion.

- Mobility issues: The wireless network solution must manage the mobility of equipments and mobility of persons in order to maintain a good connectivity. 


\subsection{IEEE-802.15.4 Standard}

Although a wireless BSN is not always a lowest duty cycle application (such as continuous ECG streaming), the ZigBee/IEEE 802.15.4 framework appears to be the most intriguing and suitable protocol suite for it. The IEEE 802.15.4 MAC offers a number of valuable ingredients for BSNs: the MAC is optimized for low power and short messages and includes peer-to-peer network support, guaranteed time slots, etc. IEEE 802.15.4 is also likely to be chosen as the radio layer basis for IEEE P1451.5-based wireless sensors [39]. Highly integrated single-chip IEEE 802.15.4-compliant transceivers are already available from a number of IC manufacturers, yet they are a bit more power hungry than simple FSK (Frequency Shift Keying) transceivers because of DSSS (Direct Sequence Spread Spectrum), but they offer better robustness and better interoperability compared with FSK. Of course, the data rate is not sufficient to carry video data in ambient applications, but it could well convey pre-processed data, e.g. from a camera system that detects when a person is moving or falling. The IEEE 802.15.4a alternate PHY may add another interesting flavour to BSNs in the not-too-distant future. Worldwide interest in ZigBee-/IEEE 802.15.4-compliant products will inspire global creativity and keep costs down. In [39], the authors present a usage scenario on wireless vital sign monitoring using IEEE802.15.4a standard. The 15.4a piconet is composed of one piconet controller (PNC) and a number of vital-sign sensors attached to the patient. The PNC, which plays the role of a data aggregator, is located at bedside; thus, the distances between the PNC and the sensors are generally shorter than 2 meters. Vital signs typically monitored in a patient's body can be categorized into two types: one type corresponds to continuous data, that is, data information (such as ECG) continuously transmitted from a sensor, and the other type corresponds to routine data, which is generated sporadically from the sensors including body temperature (BT), oxygen saturation $(\mathrm{SpO} 2)$, and blood pressure (BP). For the continuous data type, wireless transmission which supports delay QoS maintenance is required because ECG waveform is a streamed data signal.

\subsection{IEEE-802.15.4 Link Quality}

In this section, we introduce LQI (Link Quality Indicator). The LQI measurement is a characterization of the strength and/or the quality of a received packet. It can be implemented using the Energy Detection Receiver (ED Receiver), an estimation of Signal-to-noise ratio or a combination of the two methods. The use of this measure in the network and the application layers has not yet been specified in the IEEE-802.15.4 standard.

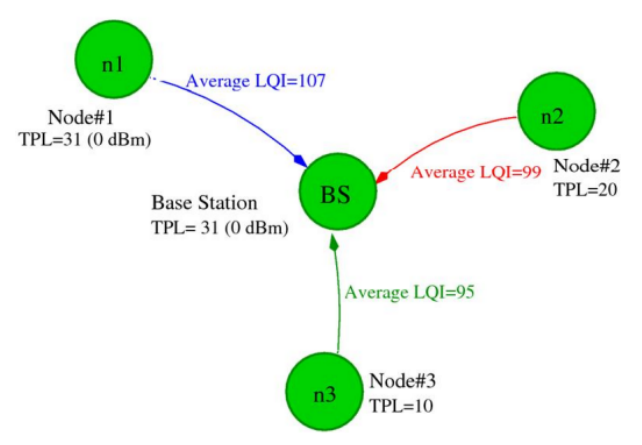

Figure 2. Sensor network with 3 nodes and a BS

LQI measurements must be performed for each received packet, and the result must be reported to the MAC layer using the original PD-DATA indication as an integer from 0-255 [39]). Minimum and maximum LQI values (0 and 255) should be associated respectively with the lowest and the highest quality of the signals that can be detected by the receiver. The LQI values are uniformly distributed within these two limits. Later on, we will see that it really depends on the type of the receiver that is used, including that of the CC2420 radio module where these limits are actually 50 and 110. Many platforms, (e.g. micaZ, Telos, Intel Mote2, ...) use a CC2420 radio module.

As a matter of fact, the LQI is a hardware indicator provided by the CC240 module, and actually is a measure of the bit error rate. In addition to the LQI, the CC2420 module also provides the RSSI (Received Signal Strength Indicator. The observed limits of the RSSI in old platforms have led several routing protocols as [40] to adopt the LQI as the preferred metric. The results given in [41] also indicate that the RSSI is strongly correlated with the PRR, except when operating at the receiver sensitivity limits. Meanwhile, the LQI can make more accurate estimates, requiring averaging many readings, which reduces flexibility and increases the cost of the estimation. The results in [41] suggest that there is a correlation between hardware indicators behavior and the protocols using them.

Experimental test. Experiments were performed using TmoteSky devices. The platform is smaller than a business card, and includes a microcontroller operating at $8 \mathrm{MHz}$, with $48 \mathrm{~K}$ of $\mathrm{ROM}$, and $10 \mathrm{~K}$ of $\mathrm{RAM}$, a $2.4 \mathrm{GHz}$ ZigBee wireless transceiver, and a USB interface for device programming and logging. Each device operates on 2 AA batteries.

In order to understand the link quality indicator we achieved some tests and shows how this parameter varies depending on the transmission power and the distance between the nodes. As shown in Fig.2, the first test involves four sensor nodes including a base station to collect the data packets. The nodes form a star 


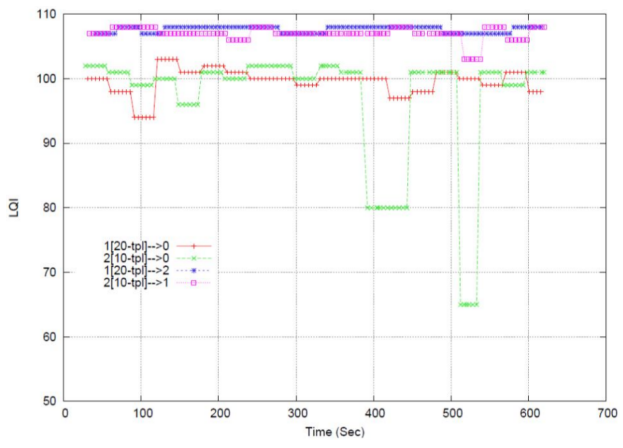

Figure 3. Link Quality vs. time and TPL

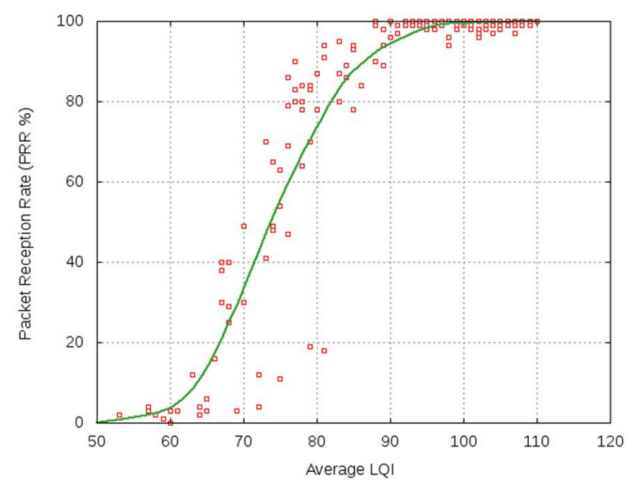

Figure 4. PRR vs. LQI. topology and use different transmission power levels (TPL). In fact, the equipment software does not directly manipulate the power in $\mathrm{dBm}$. Thus, the power level is coded by an integer between 1 and 31 corresponding respectively to $-25 \mathrm{dBm}$ and $0 \mathrm{dBm}$.

Fig. 3 plots the LQI variation over time for the network links. First, we note that LQI measurements depend on the transmission power. Indeed, the higher the transmitter power is, the better the measured LQI is. Considering the packets received by the Base Station, on average, the LQI of the link 1 -> BS is slightly higher than that of the link $2->B S$.

In the second test, we studied the LQI variation according to the distance between node 1 and the BS. The distance ranges from 3 to 35 meters and the node sends one packet every 3 seconds. The BS measures the LQI when it receives these packets. The two nodes are placed in a corridor with an ambient temperature of $20 C$. We observed the average LQI of each set of 1000 packets based on the distance between the sensor node and the BS. We also noted a variation of the average IQI in function of the distance. Generally the average LQI decreases when the distance increases.

Further measurement analysis showed that if we consider the average LQI values, the PRR follows a smooth curve suggesting a better correlation between LQI and PRR. These results are plotted in Fig.4, and the correlation coefficient (Pearson) is 0.87 . However, some cases, for instance when $L Q I=85$, could result in any value of PRR ranging from $10 \%-100 \%$. Although we did not fully understand what can cause this, we believe that it may be related to the environment changes and interferences with other network technologies such as 802.11.

Finally, in this experimental study, we show how the network links are sensitive to parameters such as the distance between the nodes and their transmission power.

\section{Satellite issues in telemedicine applications}

A satellite link raises several issues in a network deployed for telemedicine applications. Some of them may have a multifold impact on the biomedical signal in terms of: receive signal quality; signal reconstruction algorithm performance; quality of service (QoS) performance. In the following, the focus will be placed upon, (i) the satellite channel itself in relation with propagation impairments in high frequency bands, and the nature of errors occurring in a satellite link; (ii) QoS issues with a special emphasis on performance requirements related to the transmitted IP-based traffic usable in telemedicine applications, (iii) and quite significant issues related to the TCP (Transmission Control Protocol) over the satellite link. The discussion is limited to geostationary satellites.

\subsection{The satellite channel issue}

The satellite channel is a sensitive link in two respects:

(i) with regards to atmospheric impairments in high frequency bands, which can cause severe link outages;

(ii) with regards to the error behavior, bit errors tending to cluster in bursts. An analysis and a thorough characterization of error patterns are required in order to assess the impact of bit errors on higher layers protocols.

These two aspects are detailed in the following. Channel impairments in high frequency bands: In high frequency bands above the Ka band, tropospheric effects in the satellite propagation channel may be strong, and thus detrimental to communications. Two categories will be considered [19]:

Atmospheric attenuation due to gas, water vapor, clouds, the melting layer, and rain, the rain component being the prevailing factor for percentages of time lower than $1 \%$ (cf. figure 5). The rain attenuation component results in slow signal fading variations and can yield 


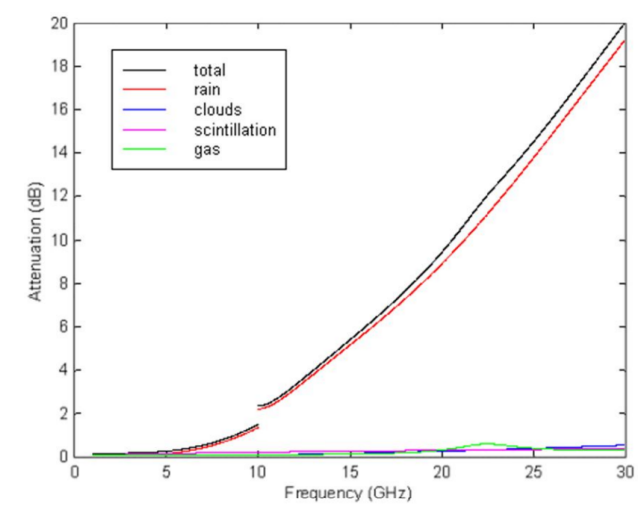

Figure 5. Components of the atmospheric attenuation versus the frequency for $0.01 \%$ of the time

a magnitude of $20 \mathrm{~dB}$ for $0.05 \%$ of an average year in $\mathrm{Ka}$ band. It represents the most serious limitation to the performance of satellite communication links in the millimeter wave domain. In addition, second order statistics of rain attenuation should also be taken into account in the design of a satellite communication system, since they directly relate to outage durations. Amplitude scintillation manifesting itself in the form of rapid signal fluctuations. Scintillation may impact the long term system availability (time percentages higher than $1 \%)$.

Channel error behavior modeling. The numerous statistical propagation models that exist today for $\mathrm{Ku}$ band and above enable to estimate the degradation on a given link in terms of signal-to-noise ratio (SNR) C/N0. Nevertheless, for new SatCom systems, C/N0 loss does not directly relate to the degradation of the QoS offered to the end user. Thus, in order to be able to obtain a sound estimation of the QoS information, it becomes necessary to resort to methods that link the channel with higher layers mechanisms or more QoS-oriented parameters. In this perspective, an analysis and a modeling of the satellite channel error behaviour are also required.

Parametric analytical models. A first approach in that direction lies upon parametric analytical models. Most of the models built for the wireless fading channel use discrete-amplitude and discrete-time Markov chains, among which the two-state Gilbert model is a wellknown one [33]. It was demonstrated that the error pattern can have a considerable impact on the performance of protocols at higher layers such as TCP or any ARQ-typed retransmission protocols [29], especially when errors occur in bursts. Combined with a broadened definition of outage events, different from the conventional definition as the exceeding of a threshold by a first order statistical parameter (such as the BER [Bit Error Rate] or the PER [Packet Error Rate] [35]), or with a peculiar framing strategy (for instance interleaving) [33], N-state Markov models enable to capture the intrinsic bit errors correlation and derive accurate predictions of the system performance when $\mathrm{N}$ increases.

Error performance methodology for ATM (Asynchronous Transfer Mode) by satellite after the ITU-R Rec. S.1062-1 WP-4B. The ITU-R Rec. S.1062-1 WP-4B constitutes a second approach towards the goal expounded above, that is, linking higher layers performance parameters to the physical layer parameters [3]. The recommendation defines a specific methodology to be applied when designing a satellite system using ATM with the purpose of satisfying the G.826 recommendation which addresses physical layer performance. The S.10621 WP-4B recommendation also links ITU I.356 QoS parameters at ATM layer with higher layers QoS parameters. It can be considered that the ITU-R S.1062-1 WP-4B recommendation provides a fruitful methodological framework for linking performance parameters located at different levels. These relations have been exploited in other contexts and studies $[7,8]$. A same type of methodology should also be applied to assess the error performance of satellite links, and their deep and maybe subtle impacts on higher layers protocols such as TCP, which will directly translates into a level of biomedical signal restitution quality in our case of interest.

(i) Errors model: In order to properly study error performance in a satellite link, a valid model of error statistics is required. The most common model is that of random errors in which a sequence of statistically independent bits is observed from two possible Bernoulli outcomes: "errored" or "non-errored". The number of errors during the observation period then follows a binomial distribution, but if the observation period is quite long and the bit error probability is very low, it can also be characterized by a Poisson distribution.

(ii) Statistical characterization of error bursts using lattice diagrams: The random errors model is inappropriate when errors occur in bursts mainly because of the memory introduced by signal processing techniques in communication systems. It is possible to statistically characterize the patterns of the errors bursts (times and interarrival times of the bursts) at the output of the decoder by means of the lattice diagram of the coder, by invoking the concept of transfer function (Viterbi) of the convolutional code, and using an algorithm that systematically and exhaustively collects all patterns of burst errors. The approach can be extended so as to characterize the effects of the scrambler, the 
descrambler, the interleaver and the concatenated coding, which are processes that are commonly used in DVB-RCS satellite communications.

(iii) Generic models of burst errors: The characterization methods presented previously are specific to a particular coding scheme. Consequently their interest is limited for globally evaluating the performance parameters of digital satellite links. Therefore more generic models are based on a small number of statistical quantities such as typically the average length $L$ of the bursts and the average number of errors per burst. Burst errors are generally assumed to follow a NeymanA contagion distribution.

\subsection{QoS issues}

For satellite communications, one of the most critical requirements is to provide the desired QoS level to the different services. The discussion will focus on IP applications. The QoS issue should be dealt with addressing the diverse network layers and the QoS architecture, and assessing the application and network behaviour. In addition to classic QoS metrics [16], subjective metrics should also be introduced in order to evaluate the IP applications from a user point of view, such as PSNR (Peak Signal to Noise Ratio) and MOS (Mean Opinion Score) [28]. The data to be transmitted is characterized mainly in terms of bit rate, overhead, error rates (BER [Bit Error Rate], or PER [Packet Error Rate]), delay, jitter, average and maximum packet sizes. In the following, two types of services are considered and their salient QoS features will be presented along with a number of constraints from higher layer protocols [20-22]: real-time applications; data-like loss-sensitive, but not or very little delaysensitive applications.

\subsection{QoS requirements for basic applications}

Real-time applications. A first typical real-time commonly required service is VoIP, which is a delaysensitive but very little loss-sensitive application. A VoIP call is expected to be intelligible. A strictly minimum bit rate of $5.3 \mathrm{kbps}$ (assuming ITU H.323 G.723.1 ACELP [Algebraic Code Excited Linear Prediction] codec) is required. It has been shown however that optimal bandwidth occupation for VoIP over satellite is around $12 \mathrm{kbps}$. VoIP bit rate also varies depending on the codec, on whether RTP (Real-Time Protocol) is compressed, and on the redundancy introduced by the headers of the protocol suite (Ethernet, IP, UDP [User Datagram Protocol], RTP). The bit rate can thus be considered to range between 5.3 and $13 \mathrm{kbps}$. A minimum MOS requirement of 3.5 ensures a good voice quality. Moreover, ITU-T G.114 Recommendation [12] specifies a maximum latency value of $150 \mathrm{~ms}$ for oneway VoIP communications. Lastly, in terms of packet corruption and loss, some experiments have shown that the satellite link is quite robust to packet corruption in clear sky or moderately degraded channel environment up to a BER of 10-5 (that is, Frame Erasure Rate or FER of 2\%) [25]. A second type of real-time services concern video applications. These applications range from real time communications to surveillance, Internet video streaming, as well as collaborative scene visualization, broadcasting and virtual meetings. Although QoS constraints are strongly dependent on the application considered, the following QoS specifications can be used as a baseline for video services [23]: the variable average bit rate allocated to a video application shall not be lower than $256 \mathrm{kbps}$ in the two ways. Critical applications such as telemedicine require a fairly good video quality. The maximum transmission delay should be lower than $400 \mathrm{~ms}$. The video codec which is used (for instance H.323) should be able to provide a good picture quality for telemedicine applications. A video connection should be established in less than $30 \mathrm{sec}-$ onds for high priority applications.

Data-like loss-sensitive, but not or very little delay-sensitive applications. In this category are for instance SMS (Short Message Service) / MMS (Multimedia Messaging System), email applications, file exchange and Internet browsing. The transmitted mean bit rate must be at least $32 \mathrm{kbps}$ for Web browsing and file exchange, and $200 \mathrm{kbps}$ for email applications [31]. BER values of up to 10-6 can be supported [31]. Moreover, the time interval between the sending of an SMS and its reception by the receiver must be between 6 and 8 seconds in average, given that actually $98 \%$ of sent SMSs are successfully delivered by a mobile user to a fixed network within a 5 sec time period, according to some telecom operators $[4,5]$. Since the integrity of SMS messages is $100 \%$, it is obvious that SMSs are well fitted to telemedicine communications, especially in emergency situations [20-22], where there is a need to transmit an alarm.

Other QoS issues. Another crucial QoS optimization method consists in properly handling and managing data traffic, especially when different services are aggregated. Differentiated services QoS architecture has received much attention these last years as traffic flows are of mixed categories (TCP flows and UDP flows for instance). By assigning each IP packet a specific traffic class, a more optimized management buffer is made possible resulting in improved bandwidth resource utilization. In particular, if excess TCP and excess UDP were both treated equally, TCP flows would reduce their rates on packet drops while UDP flows would not change, and instead monopolize the entire excess bandwidth [2]. All this leads to proper 
buffer management associated with efficient dropping strategies.

\subsection{Issues related to TCP over the satellite}

TCP is the most widely used Internet transmission protocol located at the OSI transport layer. TCP allows an end-to-end flow control mechanism between a sender and a receiver on the Internet with acknowledgments (ACKs) being sent back by the receiver to the sender, and to vary the transmission packet rate based on the rate at which acknowledgements are received back from the receiver. This mechanism enables to verify the correct delivery of data between a client and a server. TCP supports error or data loss detection, and implements a retransmission technique until the packets sent are correctly and completely received [26]. It also implements a network congestion control. All these TCP features make it a reliable and efficient transport protocol over the Internet stack, independently from the applications above it and the Internet below it. Moreover, the Internet is quite a particular network because it consists of different network topologies, bandwidth, delays and packet sizes. The satellite link possesses inherent characteristics that may have negative impacts on the TCP. Two of them are mentioned here:

- Transmission errors: satellite channels are much more prone to bit errors than typical terrestrial networks. A characterization of the burst errors in satellite links has been given in section 4 . TCP assumes that all packet drops are caused by network congestion to avoid congestion collapse [27].

- Latency: latency is due to propagation delay, transmission delay, and queuing delay [9]. Of course, the round trip time (RTT) propagation delay of about $275 \mathrm{~ms}$ in geostationary links is the prevailing term. The dominant addition to the end-to-end one way latency will be roughly $300 \mathrm{~ms}$ of fixed propagation delay [9]. This delay mainly impacts some of the TCP congestion control algorithms. Originally, the TCP protocol suite was designed for a terrestrial environment with short transmission delays that seldom exceed $250 \mathrm{~ms}$ [1]. When applied to a geostationary satellite link, TCP performs poorly due to the long latency introduced between a ground Earth station and the satellite. Latency calls for protocol-specific acceleration.

Hence, the use of TCP over the satellite raises important issues to be pondered. Two of them deserve special attention and are tackled in the following $[6,9]$.

Capacity, latency and congestion. TCP is responsible for flow and congestion control, ensuring that data is transmitted at a rate consistent with the capacities of both the receiver and the intermediate links in the network path. Since there may be multiple TCP connections active in a link, TCP is also responsible for ensuring that a link capacity is responsibly shared among the connections using it. As a result, most throughput issues are associated to TCP. Four congestion control mechanisms exist in TCP: slow start, congestion avoidance, fast retransmit before the RTO (Retransmission Time-Out) expires, fast recovery to avoid slow start [26]. The basic principle of the TCP protocol congestion mechanism can be summarized as follows [9]: a congestion window is initialized to a value of one segment upon connection startup. It determines the TCP sending rate. During the slow start phase, the congestion window doubles every round trip time (RTT), until congestion is experienced due to a data packet loss. The congestion avoidance phase is entered upon detection of congestion. Then TCP retransmits the missing segment, and the window is emptied down to its half content. If retransmitted packets happen to be lost again, the TCP sender is forced to retransmit the missing packets, but this is done with an imposed timeout where slow start is resumed and the window is reduced to one segment [26]. Consequently, the throughput becomes very low. Congestion control mechanisms in TCP thus degrade the performance of individual TCP connections over satellite links because the algorithms slowly probe the network for additional capacity, which in turn wastes bandwidth. Indeed, the satellite latency which can easily exceed $2000 \mathrm{~ms}$ is seen as evidence of a congested network or packet loss and thus TCP will not increase the rate at which it sends packets, even though there is no actual congestion or packet loss across the satellite link [1].

TCP acceleration and the security issue. Several techniques to accelerate TCP exist. Performance Enhancing Proxies (PEPs) are one of them and basically involve an alteration of the TCP header data before and after the satellite link in order to hide the high latency of the satellite link from the TCP session [1]. Examples of PEP techniques are TCP spoofing and TCP multiplexing (also known as cascading TCP or split TCP). TCP spoofing consists in shortening the delay path and thus bypassing slow start, by adding a spoofing device/software (e.g. a rooter near the satellite link) which is in charge of returning ACKs to the sender, and in the meantime suppressing the ACKs from the receiver $[1,26]$. The spoofing device also retransmits any segments lost and contains storage buffers. The TCP multiplexing technique accelerates data transfer rates across the satellite link by converting a single TCP sessions into several parallel TCP sessions. At the 
receive side, all TCP sessions are recombined into a single session [1]. Problems arise when TCP acceleration is achieved simultaneously with security by means of a VPN (Virtual Private Network) in the tunnel mode. Assuming that the data packets enter the VPN tunnel before TCP is accelerated, one is left with TCP packets entirely encrypted and the header of which cannot be altered anymore, or otherwise the authentication safeguards would be violated [1]. TCP multiplexing technique alone is compatible with VPN, but the processing must not take place inside the satellite modem. In addition, a number of performance degradation factors appear with the technique. All these performance and security issues associated with TCP acceleration have led to the development of a number of proprietary solutions to optimize the bandwidth resource and utilization for TCP over satellite. Among these, the End II End's patent-pending Broadband Network Optimization (BNO) [1] and UDcast solutions are worth to be mentioned [3]. With the revised, mobile version of the DVB-RCS standard, called DVB-RCS+M [6] as well as with the recent DVB-RCS2 standard, which both use the DVB-S2 waveform and ACM feature for the return channel, other optimization issues over a satellite link would also need to be discussed. In particular, IP encapsulation efficiency depending on the encapsulation technique employed, whether it be MPE, GSE or ULE, should be investigated for the return link as for the forward link [11].

\section{DVB-S2-based air interface}

This section describes a DVB-S2-based adaptive air interface designed by TeSA in the framework of an R\&T contract with the French Space Agency (CNES) to meet the performance constraints of bidirectional satellite communication links to be established in a post-disaster emergency situation in $\mathrm{Ku} / \mathrm{Ka}$ and $\mathrm{Q} / \mathrm{V}$ bands where strong channel impairments occur. The proposed solution allows to establish very quickly a minimum low bit rate satellite return link, using the available resources of a primary geostationary satellite system, and very small, low-cost and powerlimited dedicated ground terminals. The bi-directional transmission link is modeled within the Juzzle open source environment software with an emphasis on the return channel, deploying an adaptive strategy based on the DVB-S2 adaptive coding and modulation (ACM) scheme. This section expounds the enhanced DVBS2-like air interface proposed to support telemedicine applications in an emergency situation in a severely impaired channel environment in high frequencies; it also outlines the combined Excel/Juzzle/Matlab highlevel transmission link software simulation platform that was developed in order to assess the performance of the proposed transmission scheme. The simulator architecture follows a cross-layer approach, integrating propagation, DVB-S2-based physical layer, and traffic components. Some simulation results are also provided, emphasizing on the adopted adaptive strategy.

\subsection{System architecture and scenario}

The proposed system architecture and scenario were as follows [21]: the emergency mission signals are superimposed with those of the primary system characterized by a star topology, a classic multibeam, multicarrier, broadband bent-pipe satellite operating either in $\mathrm{Ku} / \mathrm{Ka}$ or Q/V-band, and with a 120$\mathrm{MHz}$ transponder. Thus a dedicated channel for the emergency mission is not required. The gateway has at its disposal a bandwidth of $480 \mathrm{MHz}$ (forward channel), while the user links share a total bandwidth of $240 \mathrm{MHz}$ on the return channel, in four 120$\mathrm{MHz}$ sub-bands and two polarizations, with a reuse frequency factor of 1 over 4 . Furthermore, an all-IP (Internet Protocol) architecture is assumed. For the emergency mission, a set of 4 different types of user terminals was considered: two mobile user terminals of very low transmission power (between 0.5 and 2 $\mathrm{W})$, the first one (UTA) having a patch antenna, and the second one (UTB) having a less directive antenna and thus degraded link budget performance and two deployable or transportable user terminals: UTC is a rapidly deployable âĂIJmini-gatewayâĂİ mounted on a van, transmitting at up to $50 \mathrm{~W}$, while UTD can be transported by a human user and has a transmission power of $5 \mathrm{~W}$.

\subsection{Enhanced DVB-S2 air interface}

It was proposed to adopt the ETSI DVB-S2 ACM MODCODs [25] for the return channel as it provides excellent performance close to the theoretical Shannon limit due to an advanced Forward Error Correction (FEC) scheme (concatenated $\mathrm{BCH}$ and LDPC codes), and allows an attractive waveform flexibility in presence of channel fading, with its inherent ACM capability. Incidentally, this adoption of DVB-S2 ACM schemes for the return channel was recently standardized within the DVB-RCS $+\mathrm{M}$ working group [6], but in the latter standard, very short (4 kbits) DVB-S2 PLFRAMEs were envisaged instead of normal and short lengths $(64,800$ bits for the normal frame, and 16,200 bits for the short frame). By contrast, here, standard-length DVB-S2 PLFRAMEs were assumed. In quasi-error free (QEF) environment (PER of $10^{-7}$ ), in an Additive White Gaussian Noise (AWGN) channel, DVB-S2 operates at ideal Es/N0 ranging from 16 down to $-2.35 \mathrm{~dB}$. The performance of the receiver in terms of signal acquisition/ reacquisition time, decoding thresholds, etc., all are well known [26]. The proposed novelty was the use of DVB-S2 along 
with spread spectrum and other adaptive mechanisms for return channel interactive services while DVBS2 was specifically designed for the forward link and broadcasting services. This thus raises some performance challenges to be coped with. Since the considered applications all were assumed to be IPbased ones, the DVB-S2 waveform was coupled with an efficient encapsulation mechanism, namely the GSE (Generic Stream Encapsulation) protocol at the Segmentation and Reassembly (SAR) layer, aimed at segmenting network layer IP datagrams (PDUs or Payload Data Units) into link layer DVB-S2 basic data units called BBFRAMEs (base band frames). The GSE mechanism was designed with the purpose of fully taking advantage of the innovative features of the DVB-S2, primarily in terms of reliability, flexibility, and enhanced capacity. MPE (Multi-Protocol Encapsulation) and ULE (Unidirectional Lightweight Encapsulation) have been the standard encapsulation techniques that were classically used in DVB-typed satellite systems, and as such they received abundant attention in the literature. Nonetheless, GSE constitutes a much more efficient encapsulation scheme fitted to the DVB-S2 standard in that it allows to fully exploiting the adaptive ACM capability, implementing QoS scheduling decisions and flexible placement and enhanced fragmentation of PDUs in the flow [27]. In particular, DVB-S2 GS (Generic Stream) data flows may be packetized or continuous streams. The first ones are suited to carrying PDUs of constant size, whereas the latters were designed to seamlessly adapt to input stream of any format, including continuous bit streams and variable-sized PDUs such as IP datagrams. GSE can avoid using MPEG2 packets as with MPE and ULE, which would be sub-optimal in the framework of DVB-S2. In effect, the GS flow is more suited to interactive services due to a liberty from inadequate MPEG2-TS constraints of constant bit rate and endto-end delay. In addition, due to the large sizes of a BBFRAME payload (up to 40 times as long as an MPEG2 packet), datagram fragmentation occurs less often. Measures in the Internet network backbone show that the mean size of an IP datagram is about 500 bytes, which roughly amounts to $7000 / 500=14$ IP datagrams carried in the longest available BBFRAME, against 2 or 3 fragmentations on the MPEG2-TS layer, and up to 10 in the case of ATM [27]. GS streams are tailored into 21 possible BBFRAME frames, thus offering a variety of efficiency versus error protection compromises, and predefined sizes ranging from 384 to 1,779 bytes (short BBFRAMEs), or 2,001 to 7,274 bytes (for long BBFRAMEs). Consequently, all these characteristics of GSE result in IP datagrams being delivered more rapidly, efficiently and optimally in a cross-layer perspective, with reduced redundancy and complexity [27]. A last technique deployed for the purpose of enhancing the DVB-S2 transmission performance was the Spread Spectrum (SS) in its Direct Sequence (DS) variant. Besides its resistance to interference, jamming and multipath impairments, a quite powerful property of SS exploited in the framework of this study is its processing gain $G_{P}$, defined as the ratio of the spread bandwidth over the original bandwidth in $\mathrm{dB}$. This processing gain can thus be added to the $S$ side of the SNR calculation [28]. This fruitful property is due to the power-bandwidth trade-off that exists in any radio communication system: using a spread spectrum signal enables the system to operate at negative signal to noise ratios, thus allowing to deploy smaller terminals with reduced transmission power with respect to the nonspread case. This consequently means improved battery life in the case of portable terminals, as well as an easier and quicker deployment of the terminals. Therefore the adequacy of SS is straightforward for emergency communications, in heavy rain environment [29]. The Direct Sequence Spread Spectrum (DS-SS) block can only be inserted before the base-band filter and before the modulator. In this technique, the pseudo random noise (PRN) is applied directly to the data entering the carrier modulator. The modulator therefore sees a much higher bit rate, which corresponds to the chip rate of the PRN sequence. The purpose of modulating an RF carrier with such a code sequence is to produce a directsequence-modulated spread spectrum with $((\sin x) / x)^{2}$ frequency spectrum, centered at the carrier frequency. There is no changing the point in the system where the DS-SS must be placed otherwise it would be a quite different SS technique. As a result, that means that a standard DVB-S2 transmitter cannot be used as a black box, but that the transmission chain must undergo some design adaptations, so as to conform to the provision envisaged in clause 5.1 of the mobile version of the DVB-RCS standard [6].

\subsection{Integrated simulation platform}

General description. A software model of the enhanced DVB-S2 transmission link was developed in mixed standard $C$ and Java languages within an open source software environment called Juzzle [24]. The whole simulation platform was an integrated Excel/Juzzle/Matlab software package which also implements the C DISLIN [30] data plotting library. The Juzzle simulation platform The Juzzle simulation platform is composed of two separate and standalone Juzzle components: a channel propagation time series generator component and a processing core component, and of an extern postprocessing Matlab routine.

Off-line propagation module. The propagation channel time series generation module is a pre-processing Juzzle component allowing to generate an attenuation time series configurable with a number of user parameters. 


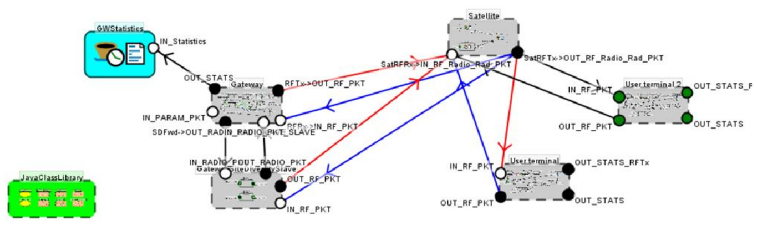

Figure 6. Juzzle link model

The synthesizer relies on the theoretical principles of the enhanced Maseng-Bakken model which was used by Lacoste [34, 35], and recently improved by Carrié. Actually, this new stochastic model enables either to stochastically interpolate initial samples to synthesize fast fluctuations of rain attenuation or to generate "on-demand" rain attenuation events starting from the three parameters: the duration of the event, the maximum attenuation value and the time position of the peak attenuation. The model improvement lies in the characterization of the conditional probability $p\left(A(t) \mid A\left(t-D t_{1}\right), \quad A\left(t+D t_{2}\right)\right)$ which enables very fast interpolation or stochastic generation of rain events "on-demand". Three of the input parameters $\left(m, s, A_{o f f s e t}\right)$ can be assessed for all link configurations using ITU-R recommended models. For the last parameter, b, a rough estimate equal to 10$4 \mathrm{~s}-1$ can be used independently of the sampling time and whatever the link considered in the North-western Europe for elevation angles between $25^{\circ}$ and $38^{\circ}$ and frequencies between $12 \mathrm{GHz}$ and $50 \mathrm{GHz}$ [36].

The Juzzle core component. The Juzzle core component is composed of 5 different nodes (Gateway, Satellite, GatewaySiteDiversitySlave, User terminal 1, and User terminal 2) as shown in Figure 3 hereafter:

This core processing module was developed in Java due to the portability, reusability, and object-oriented feature of the programming language. The module uses the Java event engine allowing to model the various delays within the system more easily. The module models the emergency satellite system at high level as shown in Figure 6, with the following nodes: two user terminals ("User terminals 1 and 2"), the master gateway, the slave gateway (to be switched to in case of strong impairments above a defined threshold when site diversity (SD) is activated) and the satellite, especially in terms of radio packet transmission, and its related link budget computation functions. As a result, the simulator is a powerful and complex integrated simulation platform handling more than 700 parameters, and being composed of 44 Java classes.

Traffic modules. Four types of traffic are modeled: VoIP, Web browsing, and SMS for the return link, and Internet aggregate traffic as an example of continuous forward link traffic. The underlying theoretical models used are:

1. for VoIP, classic ON/ OFF state machine which operates as an alternating process of talk and silence periods which are distributed according to negative exponential laws with means a- 1 and $b-1$ respectively;

2. for Web browsing traffic, the model implemented in the simulator is that of Choi and Limb [32];

3. SMS traffic is simply modeled by a generator of SMS messages whose mode of operation is as follows $[4,38]$ : $\mathrm{A}$ random number $\mathrm{N}$ from 1 to 10 (produced by a uniform random number generator) of fixed 140-byte (amounting to 160 alphanumeric characters) concatenated SMS messages is generated every period TSMS where TSMS is generated according to a negative exponential distribution.

4. the IP aggregate traffic source is modeled by a Fractional Gaussian Noise (FGN) using the Fast Fourier Transform (FFT) and Paxson's FFT algorithm. Paxson's FFT method [33] consists in synthesizing a sample path having a same power spectral density (PSD) as an FGN process. This sample path can then be used in simulations as traces of real self-similar traffic. The algorithm relies on an implementation of Paxon's selfsimilar generator written in ANSI C, and provided with in the form of a routine fft_fgn.c developed by Christian Schuler [33].

Adaptive strategy module. The adaptive strategy mechanism is at the heart of the simulator, and relies on a combination of several techniques aimed at ensuring a high availability of the transmission links in spite of severe channel impairments in the selected frequency bands (eg. about $20 \mathrm{~dB}$ in Ka band and more than $80 \mathrm{~dB}$ in $\mathrm{Q}$ band $0.01 \%$ of the time). The techniques employed are: ACM, spread spectrum with varying DSSS spread factor (L), gateway site diversity (SD) (in order to improve the downlink budget for the return channel when it is impaired by rain), and ARQ-like (Automatic Repeat Request) time diversity (TD). ARQ is only applied to SMSs, the user terminal automatically attempting at retransmitting the same SMS message at different times, until the channel conditions improve. Currently, retransmission is done on a pure deterministic basis, that is, periodically after a fixed time interval. Nevertheless, more elaborate strategies could be devised exploiting fade duration, fade slope, and inter-fade statistics so as to implement an efficient method able to predict the channel attenuation in the medium term (several minutes). For UTC and UTDtyped terminals, uplink power control (ULPC) is also 
used. It is moreover assumed that all of the user terminals of the emergency mission which are deployed over the disaster geographical area are of the same type and undergo the same ACM mode. This makes sense since the area is assumed to be quite limited in extent (less than $100 \mathrm{~km}^{2}$ which is the size of a great city such as Paris in France), which allows to consider that the link budget and transmission parameters are roughly constant over the whole area. Nevertheless, it must be kept in mind that this uniform ACM scheme over the whole area is also a consequence of a simplistic assumption concerning the channel propagation modelling, in which no spatial variability is taken into account. Only a temporal variability is modeled using the Carrié's enhancement of Lacoste's CNES/ONERA rain fading time series stochastic generator based on Maseng-Bakken model [31, 34-37].

The adaptive strategy combines ACM with spreadfactor-varying SS, the spread factor L being adapted (increased) before ACM activation depending on the attenuation level with respect to predefined thresholds, and with three different service classes being defined:

1. Premium service: VoIP, and Web browsing/SMS with a bit rate of about $128 \mathrm{kbps}$;

2. Gold service: VoIP/SMS with a bit rate of 4 to 16 kbps;

3. Basic service: SMS only with a bit rate of less than 4 kbps.

The DS-SS spread factor $\mathrm{L}$ can range from 1 to up to several thousand provided the satellite bandwidth is not exceeded. Within each service (or primary bit rate mode), the channel fluctuations are dynamically compensated by full DVB-S2 ACM MODCOD switching.

\subsection{Some results}

A number of results will be presented in this section not in an exhaustive manner, but rather selectively merely in order to illustrate the ability of the integrated simulation tool to perform relevant analyses of the performance of the low bit rate satellite communication link proposed in the context of an emergency situation and disaster management. The following configurations will be studied:

1 UTA and UTB terminals in Ku band.

2 UTD terminals in the most attenuated frequency band, that is, $\mathrm{V}$ band.

Moreover, our analysis will mainly focus on the general performance of the adaptive strategy. The normal PLFRAME (64,800 bits).
Table 1. Return link performance report for UTA and UTB terminals in Ku bandeb

\begin{tabular}{|l|l|l|}
\hline Parameters & Terminal type & $\ldots$ \\
& UTA & UTB \\
\hline Maximum uplink attenuation (dB) & 6.88 & 6.88 \\
Maximum downlink attenuation (dB) & 4.11 & 4.11 \\
Time series duration (s) & 10,800 & 10,800 \\
Used MODCODs & 15 & 15 \\
Carrier bandwidth (KHz) & 51.406 & 51.406 \\
& constant & constant \\
Maximum allowed bit rate (kbps) & 128 & 128 \\
& constant & constant \\
DS-SS factor & 1 constant & 1 constant \\
Maximum budget margin (dB) & 18.03 & 13.93 \\
Minimum budget margin (dB) & 12.22 & 8.123 \\
Mean budget margin (dB) & 15.96 & 11.861 \\
C/(N0+I0)max (dB) & 28.66 & 22.87 \\
C/(N0+I0)min (dB) & 26.61 & 24.56 \\
C/N0+I0)mean (dB) & 18.77 & 22.51 \\
Mean 1-way IP packet delay (s) & 1.03071 & 1.03071 \\
1-way IP packet delay standard & 0.14323 & 0.14323 \\
deviation (s) & & \\
Total number of ATM cells sent & 601,736 & 601,736 \\
by the terminal under study & & \\
Total number of ATM cells & 601,736 & 601,736 \\
correctly received by the gateway & & \\
Fraction of ATM cells correctly & 100 & 100 \\
received by the gateway (\%) & & \\
Transmitted throughput (kbps) & 23.627 & 23.627 \\
Correctly received throughput (kbps) & 23.627 & 23.627 \\
Mean spectral efficiency (bit/s/Hz) & 2.49 & 2.49 \\
Minimum spectral efficiency (bit/s/Hz) & 2.49 & 2.49 \\
Maximum spectral efficiency (bit/s/Hz) & 2.49 & 2.49 \\
Fraction of VoIP traffic (\%) & 40.4 & 40.4 \\
Number of VoIP ATM cells sent & 243,080 & 243,080 \\
Number of VoIP ATM cells correctly & 243,080 & 243,080 \\
received by the gateway & & \\
VoIP throughput sent (kbps) & 9.544 & 9.544 \\
VoIP throughput correctly & 9.544 & 9.544 \\
received (kbps) & & \\
Number of Web ATM cells sent & 358,656 & 358,656 \\
Fraction of Web traffic (\%) & 59.6 & $\% 59.6 \%$ \\
Number of Web ATM cells correctly & 358,656 & 358,656 \\
received by the gateway & 14.082 & 14.082 \\
VoIP throughput sent (kbps) & 14.082 & 14.082 \\
VoIP throughput correctly & & \\
received by the gateway (kbps) & 0 & 0 \\
Number of SMS ATM cells sent & 0 & 0 \\
Fraction of SMS traffic (\%) & & \\
SMS throughput sent (kbps) & & \\
SMS throughput correctly & & \\
received by the gateway (kbps) & & \\
\hline
\end{tabular}

UTA and UTB terminals in Ku band. In Ku band, UTA and UTB terminals can be used and are entirely sufficient for the defined purpose. Table summarizes the results obtained for the two terminal types using two 10,800$\mathrm{s}$ channel propagation attenuation time series. The uplink attenuation time series has a peak attenuation of $6.88 \mathrm{~dB}$ while the downlink one has a maximum attenuation of $4.11 \mathrm{~dB}$.

Figure 7 and Figure 8 show the behaviour of the adaptive mechanism for UTA and UTB terminals respectively. It can be observed that for the two 

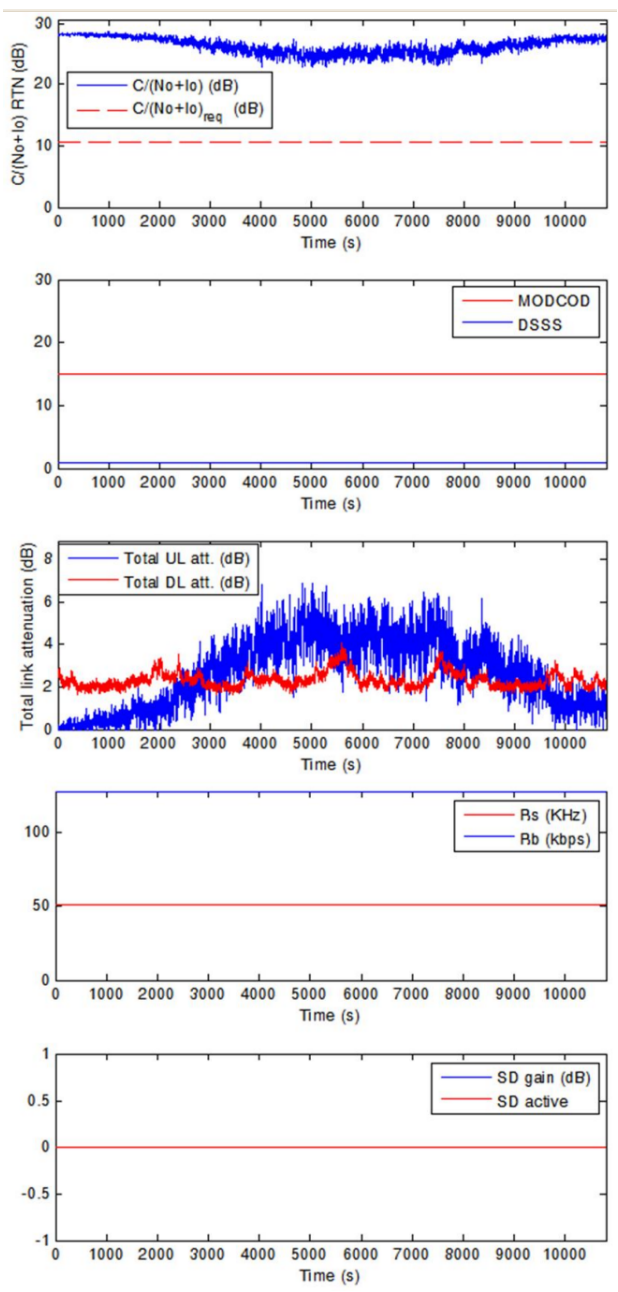

Figure 7. Adaptive strategy operation for UTA terminals in $\mathrm{Ku}$ band

types of terminal: The $6.88-\mathrm{dB}$ attenuation is totally compensated using a fixed MODCOD, namely 8 PSK $8 / 9$ characterized by a spectral efficiency of 2.49 $\mathrm{bit} / \mathrm{s} / \mathrm{Hz}$. The transmitted signal is not spread (DS-SS factor of 1). Site diversity is not required. The premium service is guaranteed with a maximum allowed bit rate of $128 \mathrm{kbps}$, which gives birth to a constant carrier bandwidth of $51.406 \mathrm{KHz}$.

UTD terminals in $\mathrm{V}$ band. In $\mathrm{V}$ band, UTA and UTB terminals cannot be used anymore. Larger terminals are required. Since UTC is known to be overdimensioned, only UTD needs to be examined here. Table summarizes the performance results obtained in a simulation with UTD terminals using two 14,160-s channel propagation attenuation time series. The uplink attenuation time series has a peak attenuation of $84.74 \mathrm{~dB}$ while the downlink one has a maximum attenuation of $37.51 \mathrm{~dB}$. It was also assumed that only a subset of the 28 DVB-S2
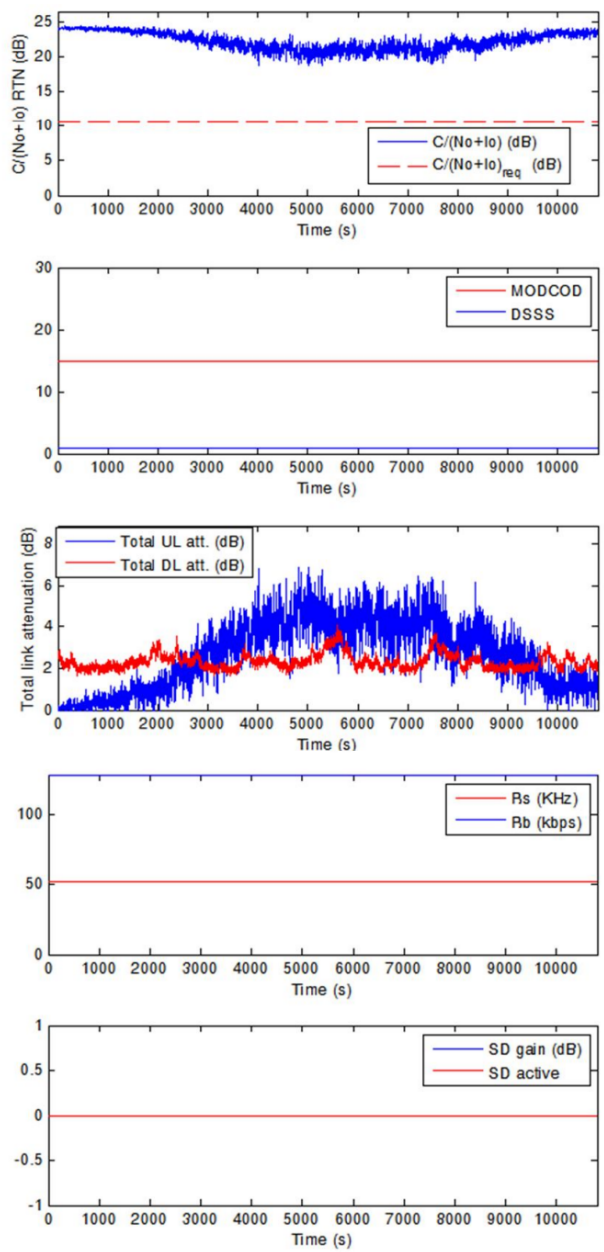

Figure 8. Adaptive strategy operation for UTB terminals in Ku band

MODCODs was used, more precisely, MODCODs 1 to 23. In other words, 32 APSK modulation was excluded.

It is worth noting that $\mathrm{SD}$ is never triggered, since the downlink attenuation never reaches the $\mathrm{SD}$ activation threshold of $64 \mathrm{~dB}$. Only the basic service (less than $4 \mathrm{kbps}$ ) can be guaranteed, due to the strong channel impairments encountered and the comparatively moderate transmission power (less than $5 \mathrm{~W}$ ) of UTD terminals. Very strong uplink attenuation values between 4,000 and $5,500 \mathrm{~s}$ lead to using the most robust MODCOD available, that is, 16 APSK 9/10 (MODCOD 23). However, even that is not sufficient to entirely compensate for the impairment, which gives birth to link outage periods. Hard activation of DS-SS can be noticed with spread factors yielding a value of more than 700 .

\section{Conclusion}

In this paper a reflection on some issues related to the use of hybrid wireless/satellite links in the 
Table 2. Return link performance report for UTD terminals in $\mathrm{V}$ band

\begin{tabular}{|c|c|}
\hline Parameters & Value \\
\hline Maximum uplink attenuation $(\mathrm{dB})$ & 84.74 \\
\hline Maximum downlink attenuation $(\mathrm{dB})$ & 37.51 \\
\hline Time series duration (s) & 14,160 \\
\hline Most used MODCODs & $\begin{array}{l}\text { QPSK } 1 / 4(92.1 \%), \\
\text { QPSK } 1 / 3(536 \%), \\
\text { QPSK } 3 / 5(1.1 \%), \\
\text { QPSK } 1 / 2(0.46 \%)\end{array}$ \\
\hline Maximum carrier bandwidth $(\mathrm{KHz})$ & 6,360 \\
\hline Minimum carrier bandwidth $(\mathrm{KHz})$ & 396.429 \\
\hline Mean carrier bandwidth $(\mathrm{KHz})$ & $2,257.678$ \\
\hline Maximum allowed bit rate (kbps) & 4 \\
\hline Minimum allowed bit rate (kbps) & 4 \\
\hline Mean allowed bit rate (kbps) & 4 \\
\hline Maximum budget margin $(\mathrm{dB})$ & 51.09 \\
\hline Minimum budget margin $(\mathrm{dB})$ & -18.75 \\
\hline Mean budget margin $(\mathrm{dB})$ & 33.25 \\
\hline $\mathrm{C} /(\mathrm{N} 0+\mathrm{I} 0) \max (\mathrm{dB})$ & 24.98 \\
\hline $\mathrm{C} /(\mathrm{N} 0+\mathrm{I} 0) \min (\mathrm{dB})$ & -50 \\
\hline $\mathrm{C} /(\mathrm{N} 0+\mathrm{I} 0)$ mean $(\mathrm{dB})$ & 6.91 \\
\hline Mean 1-way IP packet delay (s) & 1.258 \\
\hline $\begin{array}{l}\text { 1-way IP packet delay standard } \\
\text { deviation (s) }\end{array}$ & 0.976 \\
\hline $\begin{array}{l}\text { Total number of ATM cells sent } \\
\text { by the terminal under study }\end{array}$ & $1,261,175$ \\
\hline $\begin{array}{l}\text { Total number of ATM cells } \\
\text { correctly received by the gateway }\end{array}$ & $1,193,051$ \\
\hline $\begin{array}{l}\text { Fraction of ATM cells } \\
\text { correctly received by the gateway (\%) }\end{array}$ & 94.6 \\
\hline Transmitted throughput (kbps) & 37.764 \\
\hline Correctly received throughput (kbps) & 35.725 \\
\hline Mean spectral efficiency (bit/s/Hz) & 0.53 \\
\hline Minimum spectral efficiency (bit/s/Hz) & 0.5 \\
\hline Maximum spectral efficiency (bit/s/Hz) & 3.58 \\
\hline
\end{tabular}

field of telemedicine was conducted. At the starting point of our reflection, was our interest in the analysis of the performance of a combined PG-AR signal reconstruction algorithm we proposed to apply in order to remedy the problem of missing ECG samples at the user-end side. We started from the crude observation that along the transmission chain there were data errors and/or loss that could occur anywhere and anytime, and that was our motivation to investigate more thoroughly the multiple causes of such errors and loss from a pure network and telecommunication point of view. First, the context was presented with telemedicine projects (U-R-SAFE and OURSES) which enabled us to highlight some issues still open with respect to performance criteria having significant implications in health-care applications, in terms of, for example, packet error rate, energy consumption at the nodes, bandwidth occupation, etc. Furthermore, due to the multifold impact of the telecommunications and networking issues on biomedical signals, a special emphasis was laid on design constraints in a wireless network architecture, then on the satellite channel itself which can be strongly impaired in high frequency bands, and causes
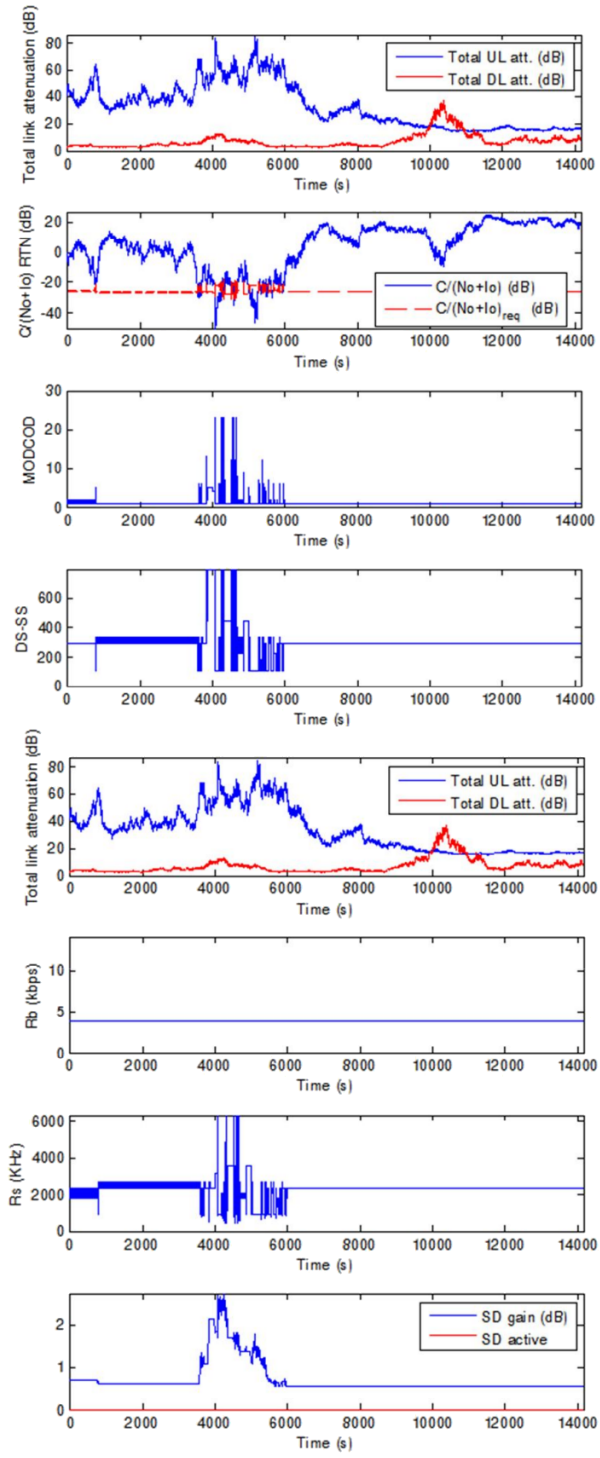

Figure 9. Adaptive strategy operation for UTD terminals in V band

errors of a particular kind. QoS issues such as that of performance requirements as to the transmitted IPbased traffic usable in telemedicine applications, and quite significant issues related to the TCP (Transmission Control Protocol) protocol over the satellite link were also surveyed. Indeed, strong propagation channel impairments requiring efficient adaptive strategies, transmission errors occurring in bursts, and high latency due to the geostationary Round Time Trip (RTT) are the three main drawbacks of the satellite path in high frequency bands. These factors combine together making data errors or packet loss very likely, which we know to be quite critical in telemedicine applications, in that human lives closely depend on high quality biomedical signal reception, and correct diagnoses. This 

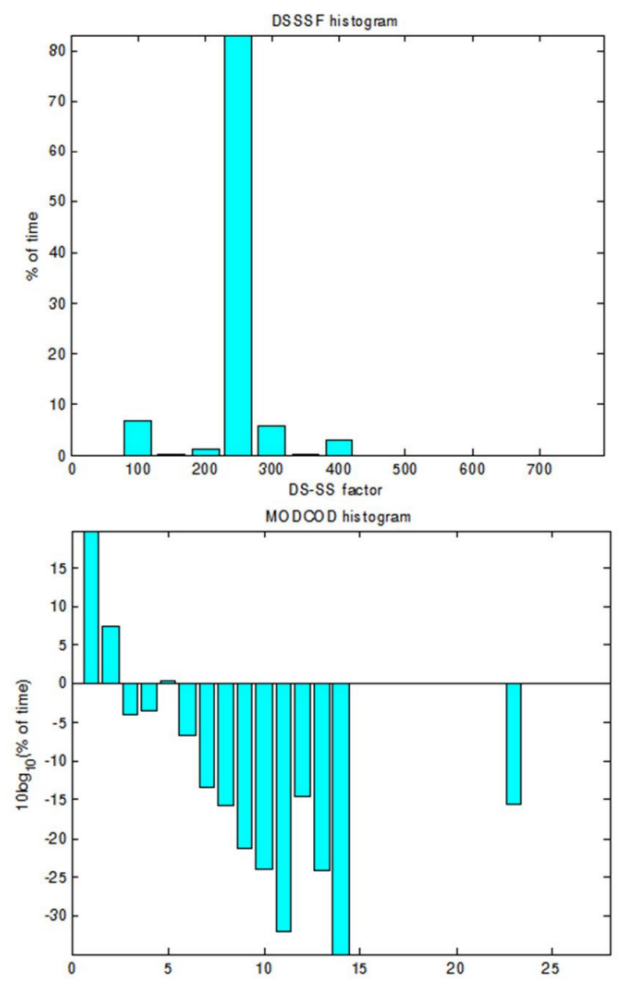

Figure 10. (a) Histogram of DS-SS factor values for UTD terminals in $\mathrm{V}$ band (left). (b) Histogram of MODCODs for UTD terminals in $\mathrm{V}$ band (right).

paper only explored a few well known networking and telecommunication issues from a qualitative point of view for telemedicine applications. The complex connections between the two worlds of telemedicine on one side, and networking and telecommunications on the other side still remain to be investigated in more details from a quantitative perspective. This will be carried out in a forthcoming paper. Last but not least, an enhanced DVB-S2-like air interface suited to telemedicine applications in an emergency situation has been described. It involves DS-SS technique and other adaptive mechanisms such power control, and site diversity for a multibeam, bent-pipe satellite system. It was designed to rapidly establish a low bit rate link for emergency communications in $\mathrm{Ku} / \mathrm{Ka}$ and $\mathrm{Q} / \mathrm{V}$ bands. Link budget analyses have shown that even though SS may be deactivated, very low transmit power user terminals UTA and UTB yield reasonable performance for the envisaged purpose up to the $\mathrm{Q}$ band, but for rather moderate channel attenuation values. This highlights the relevance and efficiency of a solution combining ACM, SS, bit reduction, and $S D$ techniques in a new adaptive strategy in order to improve the transmission performance. Such an adaptive strategy has been successfully implemented within the more general framework of an integrated Excel/Juzzle/Matlab software simulation platform designed to model the system in a high level cross-layer approach mixing propagation, physical layer, and higher layers components. Some selective results of the simulations carried out using this DVBS2-like satellite link software simulator have been partially presented highlighting its ability to perform relevant analyses of the performance of the system both at the physical layer and the network level.

\section{References}

[1] Anderson T.J, TCP/IP over Satellite: Optimization vs. Acceleration, End II End Communications, Inc. White Paper. April 2005.

[2] Durresi A, Kota S,Goyal M,Jain R and Bharani V, Achieving QoS for TCP traffic in Satellite Networks with Differentiated Services, Journal of Space Communications, Volume 17, Issue 1-3, April 2001, pp.125-136.

[3] Brandao J.C, Pinto E.L, and Maia M.A.G, A Review of Error Performance Models for Satellite ATM Networks, IEEE Communications Magazine, Broadband Satellite Network Performance, vol. 37 no. 7, pp.80-85, July 1999.

[4] ETSI, Digital cellular telecommunications system (Phase 2+); Universal mobile telecommunications system (UMTS); Technical realization of short message service (SMS) (3GPP TS 23.040 version 5.8.1 Release 5), October 2004.

[5] ETSI, TR 102444 V1.1.1: Analysis of the short message service (SMS) and cell broadcast service (CBS) for emergency messaging applications; emergency messaging; SMS and CBS, February 2006.

[6] ETSI, EN 301790 V1.5.1: Digital Video Broadcasting (DVB); Interaction channel for satellite distribution systems, May 2009.

[7] Наsнimoто A, Outage Probability Analysis in Relocatable Wireless Access Systems under Line-of-Sight Non-Rayleigh Fading, IEICE vol.E80-B no.5, pp.746-754, May 1997.

[8] Hashimoto A, Error Performance and ATM Cell Transfer Characteristics in Relocatable Wireless Access Systems, Proceedings of the IEICE, vol.E81-B, no.6, pp.1213-1223, June 1998.

[9] Henderson T. R. and Katz R. H., Transport Protocols for Internet-Compatible Satellite Networks, IEEE Journal on Selected Areas of Communications, 1999.

[10] Inigo P, Durin B, Girault N and Verelst G, OURSES: Medical assistance over Satellite, IWSSC 2008.

[11] Jegham N, Girault N, Le Guern C, Roussel G, Lohier S and Beylot A-L, VoIP over a DVB-S2 ACM link, IWSSC 2008.

[12] ITU-T, G.114 Recommendation: Transmission systems and media, general recommendations on the transmission quality for the entire Internet telephone connection: One-way transmission time, number 114 in G. ITU-T, 2000.

[13] Ganesan D, Govindan R, Shenker S, Estrin D, Highlyresilient, energy efficient multipath routing in wireless sensor networks. Mobile Computing and Communication Review, 1(2):28-36, 2002.

[14] Girault N, Jegham N, Lerouge N, Le Guern C, and BeYLot A-L, OURSES: Efficiency of IP Encapsulation over DVB-S2 Links, IWSSC 2008. 
[15] Kaсimi R, Dhaou R, and Beylot A-L, Load balancing techniques for lifetime maximizing in wireless sensor networks. Ad Hoc Networks 11(8): 2172-2186 2013.

[16] КотA S and Marchese M, Quality of service for satellite IP networks: a survey, International Journal of Satellite Communications and Networking 2003; 21:303349 (DOI: 10.1002/sat.765).

[17] Mailhes C, Castanié F, Henrion S, Lareng L, and Alonso A. The URSAFE telemedicine project: improving health care of the elderly. European Federation for Medical Informatics (MIE'03), Saint Malo, France, 2003.

[18] Mailhes C, Prieto-Guerrero A, Comet B, De Bernard $\mathrm{H}$, and Campo E. Telemedicine Applications in OURSES Project, International Workshop on Satellite and Space Communications (IWSSC'08), Toulouse, France, 2008.

[19] Ресн P. Incidence de la prise en compte des effets de techniques de mécanismes de lutte contre les affaiblissements (FMT) en bande Ka sur la gestion des ressources dans un système d'accès multimedia par satellite géostationnaire, Ph.D dissertation, Supaero, Toulouse, France, december 19, 2003.

[20] Pech P, Huang P, Bousquet M, Robert M, and Duverdier A. Simulation of an Adaptive Strategy Designed for Low Bit Rate Emergency Satellite Communications Links in $\mathrm{Ku} / \mathrm{Ka} / \mathrm{Q} / \mathrm{V}$ Bands, International Workshop on Satellite and Space Communications 2009 (IWSSC 2009), 10-11 September 2009, Siena-Tuscany, Italy.

[21] Pech P, Huang P and Bousquet M. Low Bit Rate Satellite Link for Emergency Communications, International Workshop on Satellite and Space Communications 2008 (IWSSC 2008), Toulouse, France, 1-3 October 2008.

[22] Pech P, Robert M, Duverdier A, and Bousquet M, Design and Simulation of a DVB-S2-like Adaptive Air interface Designed for Low Bit Rate Emergency Communications Satellite Link in $\mathrm{Ku} / \mathrm{Ka} / \mathrm{Q} / \mathrm{V}$ Bands, available from: http://sciyo.com/articles/show/title/designand-simulation-of-a-dvb-s2-like-adaptive-air-interfacedesigned-for-low-bit-rate-emergency-co. Chapter 9 of Satellite Communications, edited by Nazzareno Diodato, Sciyo, Rijeka, Croatia, September 2010.

[23] Ресн P, Liaison bas débit par satellite pour les situations d'urgence. Study report for work package 2, release 2.1, TeSA/CNES, 8 June 2009.

[24] Official Juzzle Web site: http://www.juzzle.org/.

[25] ETSI. EN 302307 V1.2.1: Digital video broadcasting (DVB); Second generation framing structure, channel coding and modulation systems for broadcasting, interactive services, news gathering and other broadband satellite applications, April 2009.

[26] ETSI. ETSI TR 102376 V1.1.1: Digital video broadcasting (DVB); User guidelines for the second generation system for broadcasting, interactive services, news gathering and other broadband satellite applications (DVB-S2), February 2002.

[27] Cantillo J. Codage multi-couches pour systèmes de communication par satellites, Ph.D thesis report, Télécom Paris, Toulouse, France, 19 May 2008.

[28] Ayala M.R, Gonzales E.A.A, and Franco J.J.R. Performance in QPSK and BPSK synchronous sequence DS-CDMA satellite systems, 1st International Conference on Electrical and Electronics Engineering (ICEEE), Iaghouat, Algeria,
24-27 June 2004

[29] Yoon J-H, Lee J-K, Chang D-I, and OH D-J. Spread Spectrum Technique for Digital Broadcasting System in Rain Environment, 10th International Conference on Advanced Communication Technology (ICACT), Phoenix Park, South Korea, 17-20 February 2008.

[30] Michels H, DISLIN 9.4, A Data Plotting Library, 15 October 2008. available from: http://www.mps.mpg.de/dislin/document.html.

[31] Lacoste F. Modelling of the dynamics of the Earth-space propagation channel at $\mathrm{Ka}$ and EHF bands, Ph.D thesis report, SUPAERO, Toulouse, France, September 2005.

[32] Klemm A, Lindemann C, and Lohmann M. Traffic Modeling and Characterization for UMTS Networks. Proceedings of the Globecom, Internet Performance Symposium, San Antonio TX, United States, November 2001.

[33] Paxson V. Fast, Approximate Synthesis of Fractional Gaussian Noise for Generating Self-Similar Network Traffic, Computer Communication Review, October 1997; 27(5), pp.5-18.

[34] Lacoste $F$, Bousquet $M$, Castanet L, Cornet $F$, LEMORTON J. Improvement of the ONERA-CNES rain attenuation time series synthesiser and validation of the dynamic characteristics of the generated fade events, Space Communication Journal 2005; 20(1-2).

[35] Castanet L (editor) et al. Influence of the variability of the propagation channel on mobile, fixed multimedia and optical satellite communications, SatNEx JA-2310 book, ISBN 9783-8322-6904-3, Shaker, 2008.

[36] Aroumont A, Alamanac A.B, Castanet L, Bousquet M, Cioni S, and Corazza G.E. Performance of channel quality estimation algorithms for fade mitigation techniques with $\mathrm{Ka} / \mathrm{Q} / \mathrm{V}$ band satellite systems, 9th International Workshop on Signal Processing for Space Communication, Noordwijk, Netherlands, September 2006.

[37] Lemorton J, Castanet L, Lacoste F, Riva C, Matricciani E, Fiebig U.C, Van de Kamp M, and Martellucci A, Development and validation of time-series synthesizers of rain attenuation for $\mathrm{Ka}$-band and $\mathrm{Q} / \mathrm{V}$-band satellite communication systems, International Journal of Satellite Communications and Networking, 2007; 25:575-601.

[38] ETSI. Digital cellular telecommunications system (Phase 2+); Universal Mobile Telecommunications System (UMTS); Alphabets and language-specific information (3GPP TS 23.038 v6.1.0), September 2004.

[39] IEEE Standard For Information Technology Part 15.4: Wireless LAN medium access control (MAC) and physical layer (PHY) specification for low rate wireless personal area networks (LR-WPANs), 2006.

[40] Tolle G and Culler D. Design of an applicationcooperative management system for wireless sensor networks. In Proceedings of the 2nd European Workshop on Wireless Sensor Networks (EWSN'05), pages 121_132, Istanbul, Turkey, February 2005.

[41] SRInivasan K and Levis P. RSSI is under appreciated. In Proceedings of the 3rd Workshop on Embedded Networked Sensors (EmNets'06), Cambridge, MA, USA, 2006.

[42] YANG G Z, Body Sensor Networks, Springer-Verlag London, 2006. 
[43] Prieto-Guerrero A, Mailhes C and Castanié F, Lost Sample Recovering of ECG Signals in e-Health Applications, Proceedings of the 29th Annual International, Conference of the IEEE EMBS, Cité Internationale, Lyon, France, August 23-26, 2007. 